\title{
ON STRESS FUNCTIONS FOR ELASTOKINETICS AND THE INTEGRATION OF THE REPEATED WAVE EQUATION*
}

\author{
BY \\ E. STERNBERG \\ Brown University \\ AND \\ R. A. EUBANKS \\ American Machine and Foundry Company
}

1. Introduction. The general solution due to Galerkin [1] $]^{1}$ of the field equations in classical elastostatics, was extended to elastokinetics by Iacovache [2] on the basis of a formal operational scheme originated by Gr. C. Moisil [3]. The method of derivation employed in [2], however, does not assure the completeness of the solution so obtained. In the present paper we supply a completeness proof for the generalized Galerkin solution and relate it to a generalization of Papkovich's [4] solution of the equilibrium equations. With a view toward the fact that the Galerkin vector of elastokinetics, in the absence of body forces, satifies a repeated wave equation, we prove further that every solution of such an equation may be represented as the sum of two wave functions. This result is a generalization of a theorem due to Almansi [5] regarding the integration of the biharmonic equation.

2. Completeness of the generalized Galerkin solution. The displacement equations of motion in the linear theory of homogeneous and isotropic elastic media are given by

$$
\nabla^{2} \mathbf{u}+\frac{1}{1-2 \nu} \nabla \nabla \cdot \mathbf{u}-\frac{\rho}{\mu} \frac{\partial^{2} \mathbf{u}}{\partial t^{2}}+\frac{\mathbf{F}}{\mu}=0,
$$

where $\mathbf{u}$ is the displacement vector, ${ }^{2} t$ stands for the time, $\mathbf{F}$ is the body force density, $\rho$ the mass density, while $\nu$ and $\mu$ denote Poisson's ratio and the shear modulus, respectively. Let $\mathbf{G}(P, t) b^{3}$ four times continuously differentiable for $P$ in an arbitrary region of space $D$ and $a<t<b$. Substitution into (1) confirms that every displacement field of the form

$$
\mathbf{u}=\frac{1}{2 \mu}\left[2(1-\nu) \square_{1}^{2} \mathbf{G}-\nabla \nabla \cdot \mathbf{G}\right]
$$

satisfies (1) provided

$$
\square_{1}^{2} \square_{2}^{2} \mathrm{G}=-\frac{\mathbf{F}}{1-\nu},
$$

*Received April 17, 1956. The results communicated in this paper were obtained in the course of an investigation conducted under Contract N7onr-32906 with the Office of Naval Research, Department of the Navy, Washington, D. C.

${ }^{1}$ Numbers in brackets refer to the bibliography at the end of the paper.

"Letters in boldface designate vectors; the symbols "." and " $X$ " indicate scalar and vector multiplication of two vectors, respectively. $\nabla$ is the usual del-operator.

${ }^{3}$ We shall write consistently $f(P, t)$ in place of $f(x, y, z, t), P$ being a point with rectangular cartesian coordinates $(x, y, z)$. 
where

$$
\square_{1}^{2}=\nabla^{2}-\frac{1}{c_{i}^{2}} \frac{\partial^{2}}{\partial t^{2}} \quad(i=1,2)
$$

and

$$
c_{1}^{2}=\frac{2(1-\nu)}{1-2 \nu} \frac{\mu}{\rho}, \quad c_{2}^{2}=\frac{\mu}{\rho}
$$

Equations (2), (3) constitute the solution of (1) discovered by Iacovache [2], when the body forces are absent. If $\mathbf{G}$ is independent of $t$, this solution becomes identical with Galerkin's solution [1] of the equilibrium equations." We now prove the following completeness theorem:

Let $D$, with the boundary $B$, be a bounded (not necessarily simply connected) region of space. Let $\mathbf{u}(P, t)$ be continuous in $D+B$, piecewise four times continuously differentiable for $P$ in $D$ and $-\infty<t<\infty$. If $\mathbf{u}(P, t)$ is a solution of (1), then there exists a function $\mathbf{G}(P, t)$, of the same degree of smoothness, such that the representation (2), (3) holds.

By virtue of the Stokes-Helmholtz resolution, ${ }^{5}$ there exist $\phi(P, t)$ and $\mathbf{A}(P, t)$, both having the degree of smoothness of $\mathbf{u}(P, t)$, such that

$$
2 \mu \mathbf{u}=\nabla \boldsymbol{\phi}+\nabla \times \mathbf{A} .
$$

Substitution of (6) into (1) yields, with the aid of (4) and (5),

$$
\frac{c_{1}^{2}}{c_{2}^{2}} \square_{1}^{2} \nabla \phi+\square_{2}^{2} \nabla \times \mathbf{A}+2 \mathbf{F}=0 .
$$

Next define a function $\mathbf{G}(P, t)$ by means of

$$
\mathbf{G}(P, t)=\frac{-1}{8 \pi(1-\nu)}\left[\frac{c_{1}^{2}}{c_{2}^{2}} \nabla \phi_{2}+\nabla \times \mathbf{A}_{1}\right],
$$

in which $\phi_{2}(P, t)$ and $\mathbf{A}_{1}(P, t)$ are the retarded potentials given by

$$
\phi_{2}(P, t)=\int_{D} \frac{\phi\left(Q, t-R / c_{2}\right)}{R} d \tau, \quad \mathbf{A}_{1}(P, t)=\int_{D} \frac{\mathbf{A}\left(Q, t-R / c_{1}\right)}{R} d \tau .
$$

Here $R$ is the distance from $P$ to $Q$ and the integrations are with respect to $Q$. Clearly, $\mathbf{G}(P, t)$ has the required smoothness. Moreover, from the theory of retarded potentials,

$$
\square_{2}^{2} \phi_{2}=-4 \pi \phi, \quad \square_{1}^{2} A_{1}=-4 \pi A,
$$

whence (8) implies

$$
\square_{1}^{2} \mathbf{G}=\frac{1}{2(1-\nu)} \nabla \times \mathbf{A}-\frac{c_{1}^{2}}{8 \pi(1-\nu) c_{2}^{2}} \square_{1}^{2} \nabla \phi_{2}
$$

4See also Mindlin [6] and Westergaard [7].

'See, for example, Phillips [8], p. 187.

'See Phillips [8], Chapter IX. 
By (10), (11),

$$
\square_{1}^{2} \square_{2}^{2} \mathrm{G}=\frac{1}{2(1-\nu)}\left(\frac{c_{1}^{2}}{c_{2}^{2}} \square_{1}^{2} \nabla \phi+\square_{2}^{2} \nabla \times \mathbf{A}\right),
$$

and (7), (12) imply (3).

In view of (8),

$$
\nabla \cdot G=-\frac{c_{1}^{2}}{8 \pi(1-\nu) c_{2}^{2}} \nabla^{2} \phi_{2}
$$

while (6), (11) lead to

$$
2 \mu \mathfrak{u}=\nabla \phi+2(1-\nu) \square_{1}^{2} \mathbf{G}+\frac{c_{1}^{2}}{4 \pi c_{2}^{2}} \square_{1}^{2} \nabla \phi_{2} .
$$

It follows from (14), (10), and (4) that

$$
2 \mu \mathrm{u}=2(1-\nu) \square_{1}^{2} \mathbf{G}+\frac{1}{4 \pi}\left(\frac{c_{1}^{2}}{c_{2}^{2}}-1\right) \nabla \nabla^{2} \phi_{2}
$$

and (15), (13), because of (5), yield (2). This completes the proof of the theorem.

If we define functions $\varphi(P, t)$ and $\psi(P, t)$ through

$$
\varphi(P, t)=-\nabla \cdot \mathbf{G}-\mathbf{r} \cdot \boldsymbol{\psi}, \quad \boldsymbol{\psi}(P, t)=-\frac{1}{2} \square_{1}^{2} \mathbf{G},
$$

in which $\mathbf{r}$ is the position vector of $P,(2)$ becomes

$$
2 \mu \mathbf{u}=\nabla(\varphi+\mathbf{r} \cdot \mathbf{\psi})-4(1-\nu) \mathbf{u} .
$$

On the other hand, we conclude from (16), with the aid of (3) and (4), that

$$
\square_{1}^{2} \varphi+\mathbf{r} \cdot \square_{1}^{2} \psi=0, \quad \square_{2}^{2} \psi=\frac{F}{2(1-\nu)} .
$$

If $\varphi$ and $\psi$ are independent of $t$, Eqs. (17), (18) reduce to Papkovich's [4] general solution of the equilibrium equations which was independently discovered by Neuber [9]. ${ }^{7}$

3. A theorem on the integration of the repeated wave equation. According to (3), the generalized Galerkin vector $\mathbf{G}(P, t)$ satisfies an inhomogeneous repeated wave equation. If $D$ is bounded while $\mathbf{F}(P, t)$ is continuous in $D+B$ and piecewise twice continuously differentiable for $P$ in $D,-\infty<t<\infty$, a particular solution of (3) is obtainable through an iterated retarded potential. Indeed, such a solution is given by

$$
\left.\begin{array}{l}
\mathbf{G}(P, t)=\int_{D} \frac{\mathbf{E}\left(Q, t-R / c_{1}\right)}{R} d \tau, \\
\mathbf{E}(P, t)=\frac{-1}{16 \pi^{2}(1-\nu)} \int_{D} \frac{\mathbf{F}\left(Q, t-R / c_{2}\right)}{R} d \tau,
\end{array}\right\}
$$

where $R$ is again the distance from $P$ to $Q$. We now turn to the homogeneous repeated wave equation and prove the theorem: in $[10]$.

7See also Mindlin [6], [10]. The body forces are assumed to vanish in [4] and [9]; they are included 
Let $G(P, t)$ be four times continuously differentiable for $P$ in an arbitrary region $D$ and $a<t<b$. If $G(P, t)$ is a solution of

$$
\square_{1}^{2} \square_{2}^{2} G=0, \quad c_{1}^{2} \neq c_{2}^{2},
$$

then it admits the representation

$$
G=G_{1}(P, t)+G_{2}(P, t),
$$

where

$$
\square_{i}^{2} G_{i}=0 \quad(i=1,2)
$$

and $G_{i}(P, t)$ is twice continuously differentiable in $D, a<t<b$.

It suffices to show that there exists a $G_{1}(P, t)$ which has the desired smoothness and simultaneously meets the equations

$$
\square_{1}^{2} G_{1}=0, \quad \square_{2}^{2}\left(G-G_{1}\right)=0 .
$$

Once the existence of such a $G_{1}$ has been demonstrated, we define $G_{2}(P, t)$ by

$$
G_{2}(P, t)=G-G_{1},
$$

and the proof is complete.

Equations (23), in view of (4), are equivalent to

$$
\square_{1}^{2} G_{1}=0, \quad \frac{\partial^{2} G_{1}}{\partial t^{2}}=g,
$$

where

$$
g(P, t)=\frac{c_{1}^{2} c_{2}^{2}}{c_{2}^{2}-c_{1}^{2}} \square_{2}^{2} G
$$

and, from (20),

$$
\square_{1}^{2} g=0 .
$$

Thus, we need only exhibit a function $G_{1}(P, t)$ satisfying (25), subject to (27).

Consider the function $H(P, t)$ defined by

$$
H(P, t)=\int_{0}^{t} \int_{0}^{\lambda} g(P, \xi) d \xi d \lambda .
$$

Clearly, $G_{1}=H$ conforms to the second of (25). Moreover, by (27), (28),

$$
\frac{\partial^{2}}{\partial t^{2}} \square_{1}^{2} H=\square_{1}^{2} \frac{\partial^{2} H}{\partial t^{2}}=\square_{1}^{2} g=0,
$$

whence

$$
\square_{1}^{2} H=\alpha(P)+t \beta(P) .
$$

Next, consider the function $G_{1}(P, t)$ defined by

$$
G_{1}(P, t)=H(P, t)+A(P)+t B(P),
$$


where $A$ and $B$ are particular solutions of the Poisson equations

$$
\nabla^{2} A=-\alpha, \quad \nabla^{2} B=-\beta .
$$

By virtue of (28), $G_{1}$ so defined still meets the second of (25), and according to (30), (32), it also satisfies the first of (25). Finally, $G_{1}(P, t)$ has the desired smoothness. The proof is now complete.

The foregoing theorem is the counterpart for the repeated wave equation, of Almansi's [5] theorem regarding the representability of biharmonic functions in terms of harmonic functions. A different kind of extension of Almansi's theorem was given in [11]. It should be noted that the present theorem, in contrast to those established in [5] and [11], does not involve any convexity restrictions on the region $D$.

As a consequence of the two theorems proved in this paper, and with reference to (2), (3), (4), the general solution of the equations of motion (1), in the absence of body forces, admits the alternative representation

$$
\begin{gathered}
2 \mu \mathbf{u}=\frac{\rho}{\mu} \frac{\partial^{2} \mathbf{G}_{2}}{\partial t^{2}}-\nabla \nabla \cdot\left(\mathbf{G}_{1}+\mathbf{G}_{2}\right), \\
\square_{i}^{2} \mathbf{G}_{i}=0 \quad(i=1,2) .
\end{gathered}
$$

This reduces the problem of elastokinetics to the determination of appropriate solutions of the wave equation.

\section{REFERENCES}

1. B. Galerkin, Contribution à la solution générale du problème de la théorie de l'élasticité dans le cas de trois dimensions, C. R., Acad. Sci. Paris 190, 1047 (1930)

2. Maria Iacovache, $O$ extindere a metodei lui Galerkin pentru sistemul ecuatiilar elasticitałiii, Bul. Stiint., Acad. Repub. Pop. Române A 1, 593 (1949)

3. Gr. C. Moisil, A supra sistemelor de ecuațiicu derivate parţiale lineare şi cu coeficienţi constanti, Bul. Stiint., Acad. Repub. Române A 1, 341 (1949)

4. P. F. Papkovich, Solution générale des équations différentielles fondamentales d'élasticité exprimée par trois fonctions harmoniques, C. R., Acad. Sci. Paris 195, 513 (1932)

5. Emilio Almansi, Sull' integrazione dell' equazione differenziale $\nabla^{2 n}=0$, Ann. di Mat., Ser 3, 2, 1 (1899)

6. R. D. Mindlin, Note on the Galerkin and Papkovich stress functions, Bull. Amer. Math. Soc. 42, 373 (1936)

7. H. M. Westergaard, Theory of elasticity and plasticity, Harvard Univ. Press, Cambridge, Mass., 1952

8. H. B. Phillips, Vector analysis, John Wiley \& Sons, New York, 1933

9. H. Neuber, Ein neuer Ansatz zur Lösung räumlicher Probleme der Elastizitätstheorie, Z. angew. Math. Mech. 14, 203 (1934)

10. R. D. Mindlin, Force at a point in the interior of a semi-infinite solid, Proc. First Midwestern Conf. Solid Mech., Univ. of Illinois, Urbana, Ill., 1953

11. R. A. Eubanks and E. Sternberg, On the axisymmetric problem of elasticity theory for a medium with transverse isotropy, J. Ratl. Mech. Anal. 3, 1, 89 (1954) 